\title{
THE
}

\section{A Comparative Analysis of Stationary-Phase Monte Carlo Methods}

Jimmie D. Doll

David L. Freeman

University of Rhode Island, dfreeman@uri.edu

Follow this and additional works at: https://digitalcommons.uri.edu/chm_facpubs

Terms of Use

All rights reserved under copyright.

\section{Citation/Publisher Attribution}

Doll, J. D., \& Freeman, D. L. A Comparative Analysis of Stationary Phase Monte Carlo Methods. J. Phys. Chem., 92(11), 3278-3280. doi: 10.1021/j100322a041

Available at: http://dx.doi.org/10.1021/j100322a041

This Article is brought to you for free and open access by the Chemistry at DigitalCommons@URI. It has been accepted for inclusion in Chemistry Faculty Publications by an authorized administrator of DigitalCommons@URI. For more information, please contact digitalcommons-group@uri.edu. 


\title{
A Comparative Analysis of Stationary-Phase Monte Carlo Methods
}

\author{
J. D. Doll* \\ Los Alamos National Laboratory, Los Alamos, New Mexico 87545
}

and D. L. Freeman

Department of Chemistry, University of Rhode Island, Kingston, Rhode Island 02881

(Received: August 17, 1987)

\begin{abstract}
We consider the stationary-phase Monte Carlo method and a variety of related approaches. The stationary-phase Monte Carlo method is aimed at the generic problem of performing high-dimensional integrations of rapidly oscillatory integrands. Real time numerical path integration is one important class of applications where such problems arise. We examine the relationship between the stationary-phase Monte Carlo approach and the recent work of Makri and Miller and of Filinov.
\end{abstract}

\section{Introduction}

One exciting recent development in many-body theory has been the evolution of a variety of practical numerical path integral methods for equilibrium applications. ${ }^{1}$ These path integral Monte Carlo methods are proving to be useful in the analysis of equilibrium many-body quantum phenomena, as evidenced by the rapidly increasing number of such applications. ${ }^{1,2}$

In principle, these same path integral Monte Carlo methods are applicable to problems in quantum dynamics. In practice, however, their time-dependent application has been stalled by a difficult mathematical issue. Path integral approaches involve summing contributions from all possible quantum-mechanical "paths" associated with a particular process. In equilibrium applications, the calculation of matrix elements of $\exp (-\beta H)$ (or of thermodynamic averages involving such elements) is ultimately translated by the path integral Monte Carlo approach into the problem of summing simple exponential terms, terms that are the measure of the significance of each path. In dynamical applications, however, we require matrix elements of the propagator, $\exp (i H t / \hbar)$, or, in general, the complex temperature operator ${ }^{3-6}$ $\exp (-(\beta+i t / \hbar) H)$. Such matrix elements involve not the assembly of simple exponentials, but rather the summation of complex, typically highly oscillatory exponential terms. Such a task is a difficult Monte Carlo problem. The apparent inability of Monte Carlo methods to cope directly with such phase oscillation difficulties has, in the past, led many to express a general pessimism concerning the feasibility of developing the dynamical analogue of the equilibrium path integral Monte Carlo method.

We recently introduced ${ }^{7}$ a relatively simple variation of the traditional Monte Carlo procedure aimed at overcoming the phase oscillation problems. This method was motivated in large part by a consideration of ordinary stationary-phase approaches. The aim, however, was the development of a general purpose multidimensional numerical procedure. The resulting stationary-phase Monte Carlo (SPMC) approach appears to overcome many of the difficulties outlined above. The basic idea of the SPMC approach is to use a "coarse graining" procedure to identify the regions of configuration space regions that contribute most strongly

(1) For a recent review of these methods, see, for example: Berne, B. J.; Thirumalai, D. Annu. Rev. Phys. Chem. 1986, 37, 401.

(2) For a cross section of recent applications, see, for example: J. Stat. Phys. 1986, 43, 729-1244.

(3) Miller, W. H.; Schwartz, S. D.; Tromp, J. W. J. Chem. Phys. 1983, $79,5029$.

(4) Coalson, R. D.; Freeman, D. L.; Doll, J. D. J. Chem. Phys. 1986, 85, 4567 .

(5) Doll, J. D.; Coalson, R. D.; Freeman, D. L. J. Chem. Phys. 1987, 87, 1641 .

(6) Chang, J.; Miller, W. H. J. Chem. Phys. 1987, 87, 1648.

(7) Doll, J. D.; Freeman, D. L. Adv. Chem. Phys., in press. This issue contains the conference proceedings of the Lasers, Molecules and Methods Conference, organized at Los Alamos, July, 1986. to the final result. Such an identification produces a probability distribution function suitable for use in conjunction with Metropolis importance sampling. ${ }^{8,9}$ By generating such an importance sampling function, the SPMC approach simplifies the numerical difficulties traditionally associated with Monte Carlo approaches to highly oscillatory integrands.

In the present work we analyze the basic SPMC approach in light of recent developments by Makri and Miller $^{10}$ and the related work by Filinov. ${ }^{11}$ Included is a discussion of the connection between the various approaches. We also identify practical limitations in some of the methods which are rectified in a forthcoming publication. ${ }^{12}$

\section{The Stationary-Phase Monte Carlo Method}

We begin this section by motivating the generic integrations which can be conveniently evaluated using SPMC. For notational convenience we limit the discussion to a one-dimensional system. Generalizations to many degrees of freedom are straightforward.

Key quantities in study of the quantum dynamics of manyparticle systems at finite temperatures are time correlation functions. For example, within the dipole approximation, the spectrum of a many-body system is obtained from the Fourier transform of the dipole-dipole autocorrelation function, $C_{x x}(t)$, given by

$$
C_{x x}(t)=\frac{\operatorname{Tr}\left[e^{-\beta H} x e^{i H t / \hbar} x e^{-i H t / \hbar}\right]}{\operatorname{Tr}\left[e^{-\beta H}\right]}
$$

As emphasized by Berne and Harp, ${ }^{13}$ it is convenient to consider the related autocorrelation function, $G_{x x}(t)$, given by

$$
G_{x x}(t)=\frac{\operatorname{Tr}\left[x e^{-\beta_{c}^{*} H} x e^{-\beta_{c} H}\right]}{\operatorname{Tr}\left[e^{-\beta H}\right]}
$$

where $\beta_{\mathrm{c}}$ is given by

$$
\beta_{\mathrm{c}}=\beta / 2+i t / \hbar
$$

with $\beta=1 / k_{\mathrm{B}} T, k_{\mathrm{B}}$ being the Boltzmann constant and $T$ the absolute temperature. It is easy to show that the Fourier transforms of $G_{x x}(t)$ and $C_{x x}(t)$ are related by

$$
G_{x x}(\omega)=e^{-\beta \hbar \omega / 2} C_{x x}(\omega)
$$

(8) Metropolis, N.; Rosenbluth, A. W.; Rosenbluth, M. N.; Teller, A. H.; Teller, E. J. Chem. Phys. 1953, 21, 1087.

(9) Valleau, J. P.; Whittington, S. G. In Modern Theoretical Chemistry; Berne, B. J., Ed.; Plenum: New York, 1977; Vol. 5, pp 137-168.

(10) Makri, N.; Miller, W. H. Chem. Phys. Lett. 1987, 139, 10.

(11) Filinov, V. S. Nucl. Phys, 1986, B271, 717.

(12) Doll, J. D.; Freeman, D. L.; Gillan, M. J. Chem. Phys. Lett. 1988, 143,277

(13) Berne, B. J.; Harp, G. D. Adv. Chem. Phys. 1970, 17, 63. 
Consequently, $G_{x x}(\omega)$ and $C_{x x}(\omega)$ contain the same physical information. However, $G_{x x}(t)$ has both formal and computational advantages.

By evaluating the quantum-mechanical traces in eq 2 in the coordinate representation, one obtains

$$
G_{x x}(t)=\frac{\int \mathrm{d} x \mathrm{~d} x^{\prime}\left|\left\langle x\left|e^{-\beta} c^{H}\right| x\right\rangle\right|^{2} x x^{\prime}}{\int \mathrm{d} x \mathrm{~d} x^{\prime}\left|\left\langle x\left|e^{-\beta} c^{H}\right| x\right\rangle\right|^{2}}
$$

The $x$ and $x^{\prime}$ integrations in eq 5 could be evaluated by Monte Carlo methods were the absolute square of the complex temperature density matrix elements available as the weight function. These density matrix elements are not, however, generally known analytically. These elements can be expressed in path integral form, ${ }^{14}$ a convenient starting point for numerical work. In the Fourier path integral method ${ }^{4,15-17}$ the ratio, $R$, of the density matrix element to the free particle value is expressed by

$$
\begin{aligned}
& R=\left\{\int \mathrm { da } \operatorname { e x p } \left(-\left(\sum_{k=1}^{\infty} a_{k}^{2} / 2 s_{k}^{2}+\right.\right.\right. \\
&\left.\left.\beta\langle V\rangle)+\frac{i t}{\beta \hbar}\left(\sum_{k=1}^{\infty} a_{k}{ }^{2} / 2 s_{k}^{2}-\beta\langle V\rangle\right)\right)\right\} \\
&\left\{\int \mathrm{da} \exp \left[-\left(\sum_{k=1}^{\infty} a_{k}^{2} / 2 s_{k}^{2}+\frac{i t}{\beta \hbar} \sum_{k=1}^{\infty} a_{k}^{2} / 2 s_{k}^{2}\right)\right]\right\}
\end{aligned}
$$

where

$$
s_{k}^{2}=2\left|\beta_{\mathrm{c}}\right|^{2} \hbar^{2} /\left(m \beta(\pi k)^{2}\right)
$$

where $m$ is the particle mass and $\mathbf{a}$ is the set of Fourier coefficients. The average of the potential energy evaluated along the path specified by the Fourier coefficients, $\langle V\rangle$, is defined explicitly in ref 5.

When eq 6 is introduced into eq 5 , the result is a ratio of integrations each of the generic form

$$
I(t)=\frac{\int \mathrm{d} x \rho(x) e^{i t f(x)}}{\int \mathrm{d} x \rho(x)}
$$

where $\rho(x)$ and $f(x)$ are associated with the real and imaginary parts of the action. Detailed representations for $\rho(x)$ and $f(x)$ will be given elsewhere. ${ }^{18}$ Their explicit forms are irrelevant for the current discussion. In principle, we can evaluate eq 8 by means of Monte Carlo procedures, utilizing $\rho(x)$ as an importance sampling function. For small $t$ such a procedure is a practical one. For large $t$, however, the exponential term in eq 8 will typically oscillate rapidly over distances small relative to the natural length scale of $\rho(x)$. These oscillations complicate the direct Monte Carlo evaluation of $(8)$ since they require that we include a sufficient number of quadrature points to faithfully construct all of the phase interferences. This is an unnecessary nuisance, unnecessary since these troublesome high-frequency oscillations are effectively decoupled from the weight function, $\rho(x)$, and thus cannot contribute to the integral. A complication associated with evaluating integrals of the form of eq 8 is the difficulty in formulating a procedure which smoothly interpolates between the small $t$ limit and the large $t$ (stationary-phase) limit for multidimensional integrals.

To develop a numerically useful procedure for the evaluation of integrals of the form of eq 8 , it is convenient to introduce a "damping function", $D_{\xi}(x)$, defined by

$$
D_{\epsilon}(x)=\int \mathrm{d} y p_{\epsilon}(y) \frac{\rho(x-y)}{\rho(x)} e^{i t[f(x-y)-f(x)]}
$$

(14) Feynman, R. P.; Hibbs, A. R. Quantum Mechanics and Path Integrals; McGraw-Hill: New York, 1965.

(15) Doll, J. D.; Freeman, D. L. J. Chem. Phys. 1984, 80, 2239.

(16) Doll, J. D. J. Chem. Phys. 1984, 81, 3536.

(17) Freeman, D. L.; Doll, J. D. J. Chem. Phys. 1984, 80, 5709.

(18) Doll, J. D.; Freeman, D. L.; Beck, T. L., to be published. where $p_{t}(y)$ is an arbitrary, normalized probability distribution function. In what follows we shall take $p_{\epsilon}(y)$ to be a Gaussian of width $\epsilon$. It is easy to show that eq 8 can be identically written as

$$
I(t)=\frac{\int \mathrm{d} x \rho(x) D_{\epsilon}(x) e^{i t f(x)}}{\int \mathrm{d} x \rho(x)}
$$

The effect of the introduction of $D_{f}(x)$ is to damp the integrand where the oscillations are severe. This can be seen by evaluating $D_{\epsilon}(x)$ within a gradient approximation for the special case that $\rho(x)$ is unity. When $p_{t}(x)$ is a Gaussian probability distribution function of width $\epsilon$ and $\rho(x)=1$, eq 9 is of the form

$$
D_{\epsilon}(t)=\frac{\int \mathrm{d} y e^{-y^{2} / 2 \epsilon^{2}} e^{i t[f(x-y)-f(x)]}}{\int \mathrm{d} y e^{-y^{2} / 2 \epsilon^{2}}}
$$

Expanding $f(x-y)$ through second order

$$
f(x-y)=f(x)-y f^{\prime}(x)+y^{2} f^{\prime \prime}(x) / 2
$$

produces

$$
D_{\epsilon}^{(2)}(t)=\frac{\exp \left(-(\epsilon t f)^{2} / 2\left(1-i t \epsilon^{2} f^{\prime \prime}\right)\right)}{\left[\left(1-i t \epsilon^{2} f^{\prime \prime}\right)\right]^{1 / 2}}
$$

where the superscript on $D_{\epsilon}^{(2)}(x)$ denotes the second-order gradient approximation. Analogous first-order results can be obtained from eq 13 by setting the second derivative terms to zero. The effect of the damping function can be clearly seen from eq 13. For large $t, D_{\epsilon}(x)$ is exponentially damped for points not in the stationary-phase region $\left(f^{\prime}=0\right.$ ). A numerical study of the effect of the damping function is given in ref 7 and 12. The form of eq 13 is equivalent to the procedure introduced by Filinov ${ }^{11}$ for the Monte Carlo evaluation of highly oscillatory integrands.

Although the results of integrations using $D_{\epsilon}(x)$ are formally independent of $\epsilon$, the second-order results are $\epsilon$-dependent. Furthermore, results using $D_{\epsilon}^{(2)}(x)$ are exact only in the limit that $\epsilon$ is zero. Consequently, there is a need to formulate an optimal choice of $\epsilon$ that enhances Monte Carlo convergence without introducing large systematic errors. Studies in ref 7 were limited to real values of $\epsilon$. Recently, Makri and Miller ${ }^{10}$ introduced a Monte Carlo approach to treat oscillatory integrands. This approach can be obtained from eq 13 using a particular complex, coordinate-dependent expression for $\epsilon$. In particular, if we choose

$$
\epsilon^{2}=\frac{\epsilon_{0}^{2}}{1+i t \epsilon_{0}^{2} f^{\prime \prime}}
$$

where $\epsilon_{0}$ is a real constant, and $D_{\epsilon}^{(2)}(x)$ becomes the Makri-Miller result ${ }^{10}$

$$
D_{\mathrm{MM}}(x)=e^{-(\epsilon t f)^{2}}\left[1+i t \epsilon_{0}^{2} f^{\prime}\right]^{1 / 2}
$$

The choice of $\epsilon$ in (14) serves to eliminate the imaginary parts of the second-order contributions to the exponential portion of the damping function. As discussed by Makri and Miller, ${ }^{10}$ conventional stationary-phase results for eq 10 are recovered in the large $\epsilon_{0}$ limit. Since exact results are obtained in the limit of vanishing $\epsilon_{0}$, and the stationary-phase results in the large $\epsilon_{0}$ limit, Makri and Miller ${ }^{10}$ have argued that eq 15 can be expected to be generally useful.

\section{Discussion}

We have considered here various aspects of the stationary-phase Monte Carlo method. A basic result of the present paper is the identification of the connection between our stationary-phase Monte Carlo approach and the recent methods of Filinov ${ }^{11}$ and of Makri and Miller ${ }^{10}$. This connection is summarized by eq 10 and 13-15. The SPMC method appears to offer a general tool for the analysis of a class of problems previously thought to be off limits to Monte Carlo study; the construction of high-dimensional averages of highly oscillatory integrands. Such integrals 
arise naturally in a variety of physical contexts, including the numerical path integral study of quantum dynamics. The development of the present approach and the related method, the coordinate rotation technique, ${ }^{5,6}$ suggests that progress is being made toward the development of a general Monte Carlo theory of quantum dynamics. It is likely that the methods described here will find use in other problem areas where there are difficulties associated with phase oscillations.

It is important to recognize that there are a number of potential pitfalls associated with applications of any of the approximate forms of the damping function. Perhaps the most serious is the fact that the results of integrations using an approximate damping function are not exact. There is an additional difficulty in applications of any of the second-order damping functions [eq 13 or 15]. For multidimensional integrations the second derivatives required in the evaluation of the second-order damping functions take the form of determinants. The generation of such deter- minants for many-dimensional systems may prove to be difficult. A more useful procedure may be one requiring only first derivatives which can be corrected to the exact result. Such a procedure is given in ref 12 .

Acknowledgment. We thank J. O. Hirschfelder, R. Wyatt, and R. D. Coalson for organizing the Lasers, Molecules and Methods Conference last summer at Los Alamos. That conference served as an initial sounding board for many of the ideas presented here. The timely quantum dynamics meetings organized this spring at Courant by M. Kalos and J. Lebowtiz and this summer at CECAM by M. Gillan and P. Madden were likewise of substantial assistance. Work at the University of Rhode Island was supported in part by grants from Research Corporation, the donors of the Petroleum Research Fund, administered by the American Chemical Society, and the University of Rhode Island Academic Computer Center.

\title{
Structure of Hard-Core Models for Liquild Crystals
}

\author{
Daan Frenkel \\ FOM Institute for Atomic and Molecular Physics, P.O. Box 41883, 1009 DB Amsterdam, The Netherlands \\ (Received: July 16, 1987; In Final Form: October 28, 1987)
}

\begin{abstract}
The results of recent computer simulations on fluids of nonspherical hard-core particles are discussed. New data are presented on the structure and dynamics of a system of hard spherocylinders with length-to-width ratio $L / D=5$. These data show that such spherocylinders can occur in at least four stable phases, viz., isotropic fluid, nematic liquid crystal, crystalline solid, and, surprisingly, a smectic A phase.
\end{abstract}

\section{Introduction}

Computer simulations of classical many-body systems can be used to gain insight in the microscopic behavior of real liquids and solids. Two distinct, and often complementary, approaches may be distinguished. On the one hand, one may carry out simulations on a realistic models in order to assist the interpretation of real experiments. On the other hand, computer simulations on idealized models of dense phases may be used to test theoretical concepts. Occasionally, computer simulations on simple model systems have yielded results that were qualitatively different from what was expected on the basis of the theories current at the time. A prime example of such a "computer discovery" is the observation by Alder and Wainwright of the long-time tails in the velocityautocorrelation function of hard spheres. ${ }^{1}$ Our current theoretical understanding of simple liquids is, to a large extent, based on the results of such simulations.

The situation is different for more complex fluids, such as liquid crystals. For the latter class of materials, direct comparison between experiment and simulation is difficult, because such simulations are very time-consuming (although not impossible; see ref 2). Simulation of idealized models for liquid crystals is also less than straightforward because there is no consensus as to what constitutes an "ideal" liquid crystal.

For atomic liquids it is well-known that the structure of the fluid is almost completely determined by the short-range repulsive forces acting between the atoms. In fact, the success of the hard-sphere fluid as a reference system in thermodynamic perturbation theories for simple liquids ${ }^{3,4}$ is largely a consequence of this fact. In contrast, it is at present not known whether the structure of more complex liquids, such as liquid crystals, is also

(1) Alder, B. J.; Wainwright, T. E. Phys. Rev. A 1970, 1, 18-21.

(2) Picken, S. J. Internal Report; University of Groningen, 1984.

(3) Barker, J. A.; Henderson, D. J. Chem. Phys. 1967, 48, 4714.

(4) Weeks, J. D.; Chandler, D.; Andersen, H. C. J. Chem. Phys. 1971, 54, 5237 . primarily determined by excluded volume effects. From the theoretical work of Onsager ${ }^{5}$ we know that a fluid of (infinitely) thin spherocylinders with length $L$ and diameter $D$ must undergo a transition from the isotropic to the nematic phase at a number density of order $1 /\left(L^{2} D\right)$. At this density the fraction of the volume occupied by the spherocylinders is still vanishingly small (of order $D / L$ ). Recent computer simulations on hard ellipsoids-of-revolution with more realistic shapes ${ }^{6,7}$ have shown that a stable nematic phase is possible for this class of hard-core molecules if the length-to-breadth ratio is either larger than 2.5 or less than $0.4{ }^{8}$ These results do not yet imply that nonspherical hard-core interactions are the cause of orientational order in real nematic liquid crystals. In fact, two additional factors are often invoked to explain the stability of nematic liquid crystals, namely, (1) long-range anisotropic forces ${ }^{9}$ which tend to induce orientational order and (2) the presence of flexible tails attached to the rigid molecular core. ${ }^{10}$ The effect of the flexible tails is to stabilize the liquid phase with respect to the crystalline solid. However, now that we know that hard spheroids, that have neither longrange interactions nor flexible tails, form a nematic phase over a rather wide range of length-to-breadth ratios, we can begin to test thermodynamic perturbation theories. Such tests should enable us to ascertain whether nonspherical hard-core fluids can serve as "reference" systems for nematic liquid crystals in the same way that the hard-sphere fluid is a reference system for, say, liquid argon.

\footnotetext{
(5) Onsager, L. Ann. N. Y. Acad. Sci. 1949, 51,627

(6) Eppenga, R; Frenkel, D. Mol. Phys. 1984, 52, 1303

(7) Frenkel, D; Mulder, B. M. Mol. Phys. 1985, 55, 1171

(8) Pioneering simulations of hard spherocylinders with $L / D=2$ were carried out by Vieillard-Baron Mol. Phys. 1974, 28, 809. However, in that system Vieillard-Baron did not observe nematic ordering.

(9) Maier, W.; Saupe, A. Z. Naturforsch., A: Astrophys., Phys., Phys. Chem. 1958, $13 A, 564$.

(10) Dowell, F.; Martire, D. E. J. Chem. Phys. 1978, 68, 1088. Ibid. 1978, 68, 1094. Ibid. 1978, 68, 2322. Dowell, F. Phys. Rev. A 1983, 28, 3526.
} 